\title{
Combining Powder Formulations of Drugs with Food and Beverages to Improve Palatability
}

\author{
Shimako Tanaka, Shinya Uchida,* Mai Sotoyama, Yasuharu Kashiwagura, and Noriyuki Namiki \\ Department of Pharmacy Practice \& Science, School of Pharmaceutical Sciences, University of Shizuoka; \\ 52-1 Yada, Suruga-ku, Shizuoka 422-8526, Japan. \\ Received June 10, 2020; accepted September 15, 2020
}

The taste of medicines can significantly affect patient adherence. Pediatric patients often cannot take powder medicines because of their unpleasant taste. Therefore, patients' parents and health care professionals, including pharmacists, often combine medicines with food or beverages to make them easier for pediatric patients to consume because this can reduce their unpleasant taste. The purpose of this study was to evaluate the palatability of powder formulations of azithromycin and carbocysteine and explore their combination with food or beverages to improve palatability for pediatric patients. We quantitatively evaluated the palatability of powder formulations by performing the gustatory sensation test using the visual analog scale score. The gustatory sensation tests were performed on 16 healthy adult volunteers (age 23.0 \pm 2.6 years) and indicated that some food and beverages improved the palatability of the powder formulations of azithromycin and carbocysteine. The results of this study indicate that ice cream improves the palatability of azithromycin, while yogurt improves the palatability of carbocysteine. Moreover, the subjects recommended these same combinations for pediatric patients. This study suggests that some foods and beverages improve the palatability of powder formulations, thereby decreasing the possibility that pediatric patients will refuse medications because of their unpleasant taste.

Key words powder formulation; adherence; palatability; pediatric patient; food; beverage

\section{INTRODUCTION}

There is a general acceptance of the benefits of solid dosage forms in oral administration over liquid dosage forms in terms of stability, dosing and administration issues. ${ }^{1-4)}$ For pediatric patients who are unable to swallow capsules or tablets, liquid formulations, including solutions, syrups, suspensions and emulsions, are most appropriate. For substances that are unstable or cannot be taste-masked in liquid preparations, powders or multiparticulate formulations (e.g., beads, granules and mini tablets) are encouraged. ${ }^{5)}$

Adherence is a major issue in determining the success of therapy in pediatric patients. ${ }^{6,7)}$ Pediatric patients often refuse syrup or powder formulations because of their unpleasant taste. Drugs with an unpleasant bitter taste are generally difficult to swallow for pediatric patients, resulting in poor adherence to treatment regimens and consequently reduced drug efficacy. ${ }^{8,9)}$ Furthermore, it has been reported that parents want to see improvements in a variety of factors, including the safety of medications, a reduction in the number of doses required each day and taste and smell. ${ }^{10)}$

The bitterness of active pharmaceutical ingredients is one of the major challenges faced by pharmaceutical scientists formulating oral dosage forms. Furthermore, the International Council for Harmonisation of Technical Requirements for Pharmaceuticals for Human Use Guidelines indicate that orally administered pediatric medicines must be palatable to ensure dose acceptance and regimen adherence. In addition, alternative dose administration strategies should be considered for pediatric populations who cannot be accommodated by the intended dosage form. ${ }^{11)}$ The guidelines recommend that these can be dosed directly into the mouth of the pediatric patient, by mixing the prescribed dose with a small amount of soft food or with a drink prior to administration. ${ }^{5)}$ In such cases, these formulations are often combined with food and beverages to improve palatability in clinical settings. Although the palatability of drugs affects adherence and thus the achievement of the desired therapeutic effect, few studies have been reported. In addition, there are limited quantitative studies on the improvements in the acceptance of medicines when combined with food and beverages.

Azithromycin is a potent macrolide antibiotic and is used long-term in patients with chronic inflammatory lung diseases, such as cystic fibrosis. ${ }^{12}$ Carbocysteine is conventionally prescribed as a mucolytic agent because of its capacity to reduce mucus viscosity by modifying the biochemical properties of mucus. ${ }^{13)}$ These drugs are commercially available as powder formulations for pediatric patients. The purpose of this study was to evaluate the palatability of powder formulations and explore their combination with food or beverages to improve palatability for pediatric patients.

\section{MATERIALS AND METHODS}

Test Formulations, Food and Beverage All test formulations were kindly donated by Towa Pharmaceutical Co., Ltd. (Osaka, Japan). Powder formulations of azithromycin (powder $10 \%$ ) and carbocysteine (dry syrup 50\%) were used. Water (Natural Mineral Water, Suntory, Tokyo, Japan), barley tea (Kenko-mineral Barley Tea, Ito-en, Tokyo, Japan), oolong tea (Suntory Oolong Tea, Suntory), orange juice (Nacchan-orange, Suntory), sports drink (Aquarius, Coca-Cola Japan, Tokyo, Japan), yogurt (Meiji Tokachi Yogurt, Meiji Dairies Corporation, Tokyo, Japan) and ice cream (Häagen-Dazs Vanilla, Häa- 
gen-Dazs Japan, Tokyo, Japan) were obtained commercially.

Gustatory Sensation Tests To evaluate the taste of powder formulations, gustatory sensation tests were performed on 16 healthy adult volunteers (age 23.0 \pm 2.6 years). All subjects provided written informed consent, and the study protocol was approved by the Ethics Committee of the University of Shizuoka, Japan.

A randomized, crossover design was used to assess sensations while taking azithromycin and carbocysteine with water and four groups of beverages (barley tea, oolong tea, orange juice, and sports drink), followed by yogurt and ice cream (Fig. 1). Before the start of the trial, one packet of powder formulations $(1 \mathrm{~g})$ of azithromycin $(100 \mathrm{mg})$ and carbocysteine $(500 \mathrm{mg})$ were mixed with each food $(5 \mathrm{~g})$ and beverage $(5 \mathrm{~mL})$. The subjects evaluated the bitterness and overall palatability of the mixture while holding it in their mouth for $30 \mathrm{~s}$ (the first evaluation) using a visual analog scale (VAS) with a maximum value of $100 \mathrm{~mm}$. They evaluated again $30 \mathrm{~s}$ after spitting the mixture out of the oral cavity (the second evaluation). In addition, subjects used VAS scores to rate how highly they would recommend each combination for use in pediatric patients.

Statistical Analyses All data are presented as the median. All statistical analyses were performed using GraphPad Prism

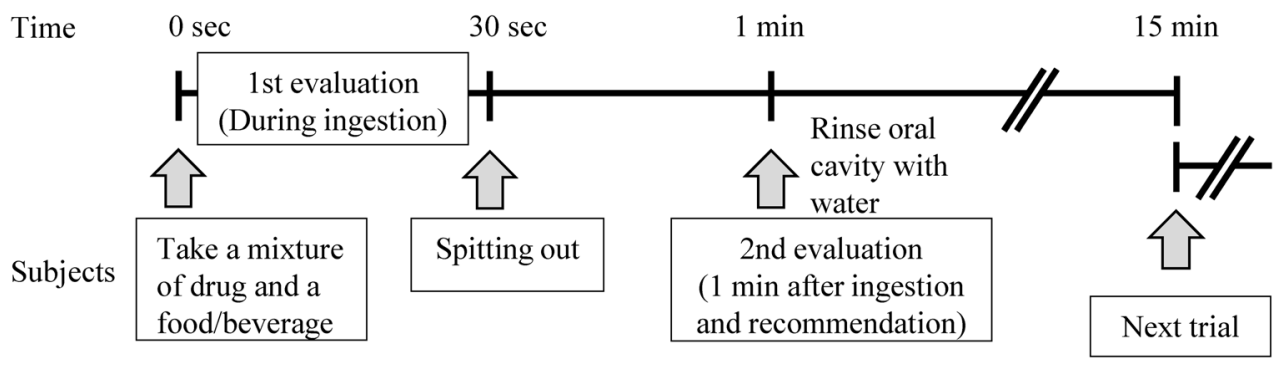

Fig. 1. Schedule of the Gustatory Sensation Test for the Powder Formulations with Food and Beverages

(a)

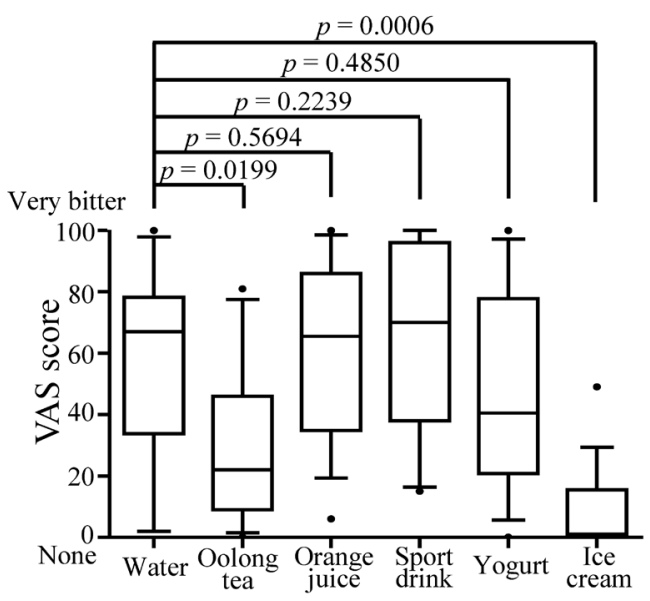

(c)

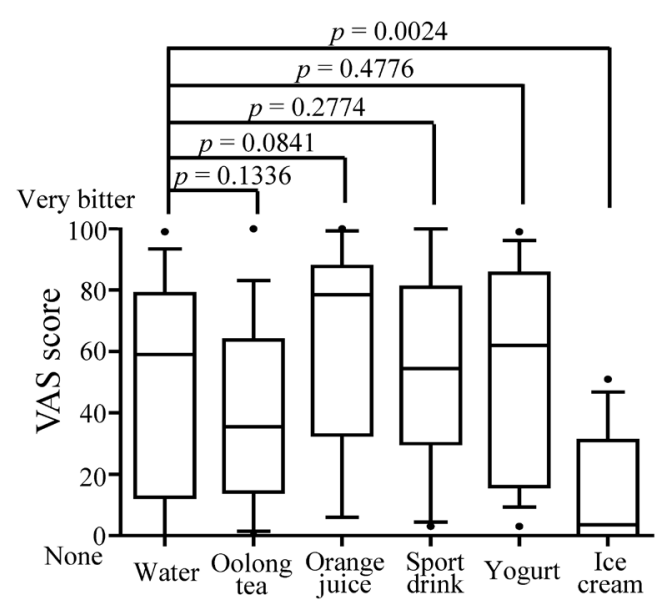

(b)

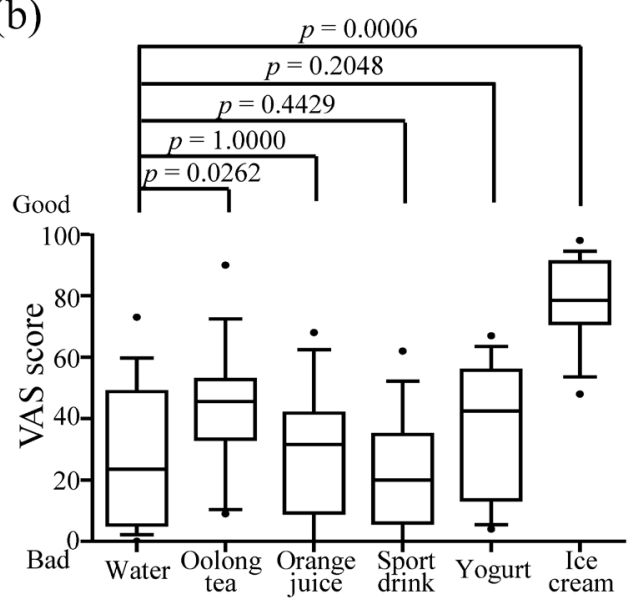

(d)

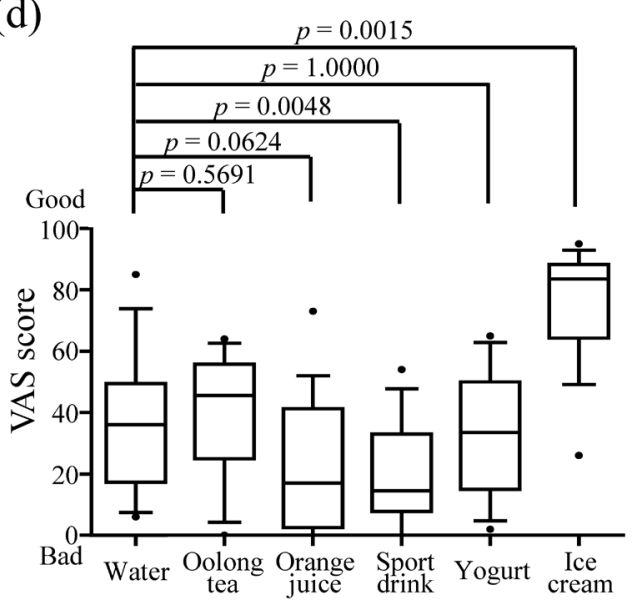

Fig. 2. The VAS Scores of Bitterness (a, c) and Overall Palatability (b, d) of the Azithromycin Powder Formulation Taken with Food and Beverages at the First Evaluation $(a, b)$ and Second Evaluation (c, d) on in the Gustatory Sensation Test

In this box plot, the top, middle and bottom represent the 75 th percentile, median and 25 th percentile, respectively. Error bars represent the 90 th and 10 th percentiles, and each point represents an outlier. First evaluations are during ingestion, and second evaluations are 1 min after intension. Nonparametric testing by the Wilcoxon signed-rank test with Bonferroni's correction was used to detect significant differences among each food and beverage for each test compared with water. 
(version 5.02; GraphPad Software, San Diego, CA, U.S.A.) For comparisons with water, the Wilcoxon signed-rank test with Bonferroni's correction was performed. Differences were considered statistically significant when the $p$ value was less than $0.01(=0.05 / 5)$.

\section{RESULTS AND DISCUSSION}

In clinical settings, powder or granule formulations are often administered to pediatric patients in combination with food and beverages to improve their palatability. ${ }^{5)}$ Nevertheless, the palatability was not evaluated using gustatory sensation test. So, we quantitatively evaluated the palatability of powder formulations by performing gustatory sensation tests using VAS scores and investigated taste to identify food and beverages that could be combined with powder formulations to improve the sensations experienced when taking the drug. Previous studies showed that using the VAS score in human gustatory sensation tests is an appropriate method to quantitatively evaluate the palatability of several oral formulations. ${ }^{14-19)}$ VAS is widely used for assessing the levels of pain or palatability in human subjects and is regarded as a reproducible approach that provides a good representation of subjective measurements. ${ }^{20)}$

(a)

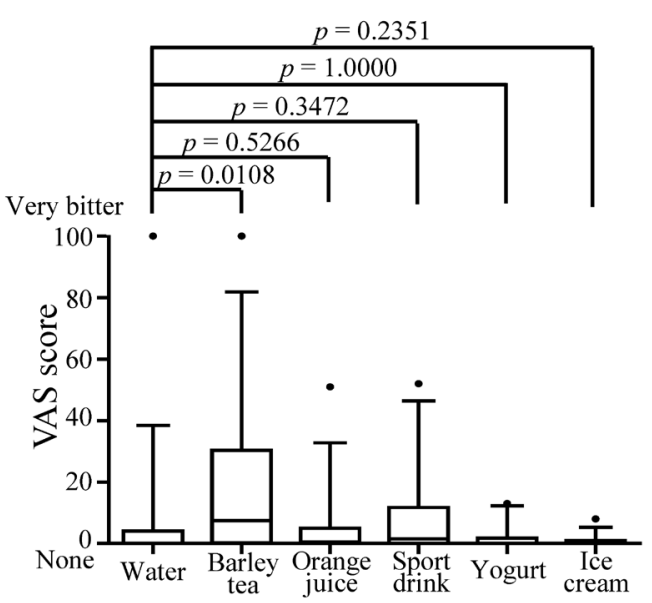

(c)

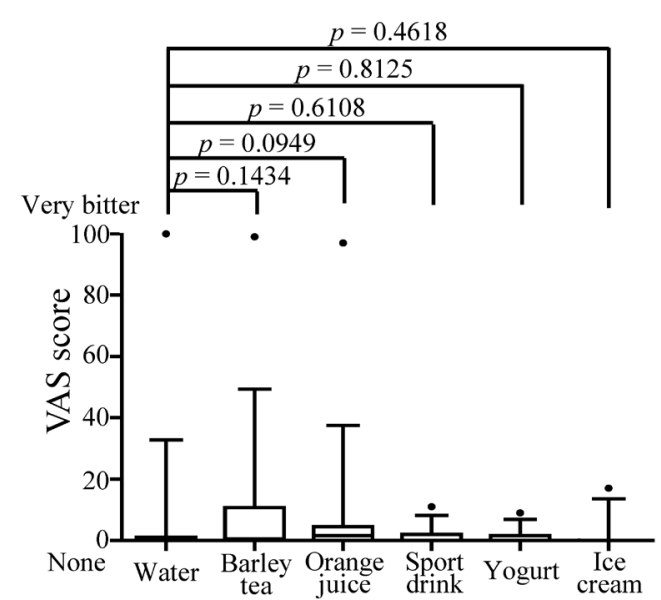

The median VAS scores for the bitterness of azithromycin in the first and second evaluations are shown in Figs. 2a and c, respectively. The VAS scores of azithromycin taken with water in the first and second evaluations were 67 and 59, respectively, suggesting that the bitterness of azithromycin remained in the mouth for at least approx. $30 \mathrm{~s}$ after spitting out, or in other words, after the patient swallowed the powder formulation. The VAS scores for the bitterness of azithromycin significantly decreased to 1.0 compared with water when taken in combination with ice cream $(p=0.0006)$. In the second evaluation, the VAS scores for the bitterness of azithromycin decreased to 3.5 ( $p=0.0024)$, suggesting that ice cream could suppress the bitterness of azithromycin. In general, diffusion coefficient of drug in the saliva juice around taste buds in human oral cavity becomes smaller in high-viscosity solution. $^{21)}$ Ice cream had high viscosities than beverages and expected to have great effect of taste masking.

Similarly, the VAS scores in the first and second evaluations decreased (22 and 35.5, respectively) when combined with oolong tea, while those values increased (65.5 and 78.5, respectively) when combined with orange juice. In a previous study, Uchida et al. reported that acidic beverages should be avoided when taking powder formulations of macrolide antibiotics because the acidic beverage was found to produce
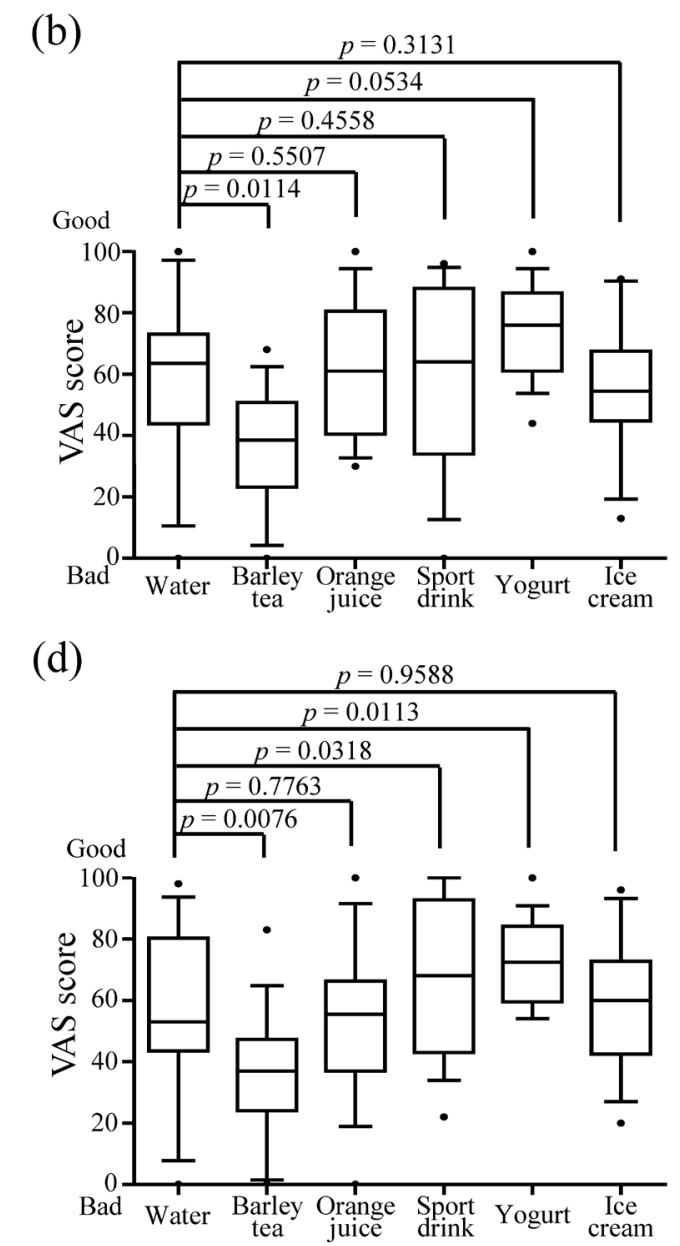

Fig. 3. The VAS Scores of Bitterness (a, c) and Overall Palatability (b, d) of the Carbocysteine Powder Formulation Taken with Food and Beverages at the First Evaluation (a, b) and Second Evaluation (c, d) in the Gustatory Sensation Test

In this box plot, the top, middle and bottom represent the 75th percentile, median and 25th percentile, respectively. Error bars represent the 90 th and 10 th percentiles, and each point represents an outlier. First evaluations are during ingestion, and second evaluations are 1 min after intension. Nonparametric testing by the Wilcoxon signed-rank test with Bonferroni's correction was used to detect significant differences among each food and beverage for each test compared with water. 
Table 1. VAS Scores of Recommendations of the Combinations for Use in Pediatric Patients

\begin{tabular}{|c|c|c|c|c|}
\hline Food/beverage & Azithromycin (25th, 75th percentiles) & $p$-Value & Carbocysteine (25th, 75 th percentiles) & $p$-Value \\
\hline Water & $50.0(10.0,60.3)$ & - & $57.5(37.3,81.0)$ & - \\
\hline Barley tea & - & - & $40.0(12.5,58.3)$ & 0.0016 \\
\hline Oolong tea & $50.0(20.0,54.5)$ & 0.776 & - & - \\
\hline Orange juice & $7.0(0,22.8)$ & 0.012 & $68.0(47.5,80.8)$ & 0.293 \\
\hline Sports drink & $17.0(0.25,22.0)$ & 0.020 & $62.5(49.8,94.8)$ & 0.047 \\
\hline Yogurt & $26.0(12.8,49.8)$ & 0.315 & $80.0(64.5,90.5)$ & 0.004 \\
\hline Ice cream & $92.0(80.3,98.5)$ & 0.001 & $66.0(49.8,78.5)$ & 0.552 \\
\hline
\end{tabular}

Data represent the median $(n=16)$. A paired $t$-test with Bonferroni's correction was used to detect significant differences among the food and beverages for each test compared with water.

a strongly bitter taste, as stated in the information sheet for Clarith $^{\circledR}$ dry syrup. ${ }^{22,23)}$ Because azithromycin also belongs to the macrolide antibiotic class of medicines, orange juice might increase the bitterness of azithromycin with the same mechanism. In our study, the sport drink ( $\mathrm{pH} 3.5)$ and orange juice ( $\mathrm{pH}$ 3.6) were mild acidic solutions, the other drinks were in a neutral range $(\mathrm{pH}=6.0-7.5)$. According to data of dissolution testing, the dissolved rate of azithromycin formulation in $\mathrm{pH}$ 1.2 and 6.8 at $5 \mathrm{~min}$ were 97.9 and $20.7 \%$, respectively. This result indicates that mild acidic drinks seem to enhance release of azithromycin caused by the dissolution of the alkaline polymer film-coating.

The median VAS scores for the bitterness of carbocysteine in the first and second evaluations are shown in Figs. 3a and c, respectively. The VAS scores for the bitterness of carbocysteine taken with water in the first and second evaluations were both 0 . The VAS scores when carbocysteine was combined with food and beverages except barley tea were similar $(0-1.5)$, but those of barley tea in the first evaluation were higher.

Overall palatability can be defined as an overall assessment, including five tastes and mouthfeel. The VAS scores for the overall palatability of azithromycin taken with water in the first and second evaluations were 23.5 and 36 , respectively (Figs. 2b, d). The VAS scores for the overall palatability of azithromycin significantly increased to 78.5 and 83.5 in the first and second evaluations, respectively, when taken in combination with ice cream. When taken with oolong tea, the VAS scores also increased to 45.5. In contrast, VAS scores for palatability decreased when azithromycin was taken with yogurt and a sports drink. For carbocysteine, the VAS scores for overall palatability when taken with water in the first and second evaluations were 63.5 and 53, respectively (Figs. 3b, d). Compared with water, the VAS scores for overall palatability in the first and second evaluations increased to 76 and 68 when combined with yogurt but decreased to 38.5 and 37 , respectively, when combined with barley tea. As for other food and beverages, there were no significant differences compared with water. Overall palatability can be defined as an overall assessment, including five tastes and mouthfeel. The improvement of the palatability of powder formulations of azithromycin considered to be due to a combination of several factors. First, the VAS scores for the bitterness of azithromycin significantly decreased compared with water when taken in combination with ice cream, demonstrating that the ice cream successfully masks the unpleasant taste of azithromycin and improve the palatability. In addition, other factors; its sweet flavor and smooth velvety texture improve the palat- ability. Cooling effect of ice cream also aids in reducing the bitterness.

The subjects used VAS scores to rate how likely they would recommend each combination for use in pediatric patients after the second evaluation (Table 1). Azithromycin showed the highest VAS scores when combined with ice cream, and carbocysteine showed the highest VAS scores when combined with yogurt. The powder formulation of azithromycin has unpleasant palatability. However, the roughness, bitterness and astringency might be suppressed, and the overall palatability and subject recommendations significantly improved when azithromycin and ice cream are combined. Although oolong tea improved the overall palatability of azithromycin, the subjects' recommendation for use in pediatric patients was at the same level as for water. Therefore, subjects did not recommend the combination with oolong tea, which itself is bitter. The VAS score of subject recommendations for use in pediatric patients when combined with barley tea was lower than the combination with water, indicating combinations with carbocysteine and barley tea should be avoided for pediatric patients. The roughness and bitterness of carbocysteine might be suppressed by yogurt, although bitterness and astringency were intensified, and sweetness was suppressed by barley tea.

Previously, some studies were reported to evaluate the bitterness of some medicines quantitatively using a taste sensor. $^{21-25)}$ The research of taste sensors has made a breakthrough by achieving high selectivity for each taste, especially bitterness. For example, Tanigake et al. evaluated the bitterness intensity of clarithromycin with some beverages by a taste sensor. ${ }^{22)}$ They found that co-administration with an acidic sports drink was the most bitter using either gustatory sensation tests and the taste sensor. Interestingly, they indicated that the taste was high correlation with human sensory score. However, the study was not exploring the combination with food or beverages to "improve palatability." In addition, they did not mention that how the taste sensor detects components which enhance or suppress the bitterness of clarithromycin. Previously, our group also investigated the palatability of the physical and organoleptic taste-masked orally disintegrating tablet of famotidine and amlodipine, and evaluated the predictability of human gustatory sensation by the a-Astree electronic gustatory test. ${ }^{14)}$ Although electronic gustatory systems can reduce potential ethical concerns about taste assessments of medicinal products as the absolute number of samples used in human gustatory sensation tests may be reduced, the prediction of palatability using electronic gustatory systems cannot replace human gustatory sensation tests. Especially overall palatability is including taste/after taste, 
somatosensory modalities such as touch (texture), grittiness, astringency, temperature, cooling effect, appearance even sound and smell. Smoothness, temperature and texture of ice cream and yogurt might be factors for good palatability. For testing in the taste sensor, compounds must be each dissolved and diluted in water to form solutions. Then the taste sensor is difficult to evaluate the overall palatability of solid, in short mixtures of powder formulation and food. We evaluated the palatability of the formulations in a clinical study. Although there are an enormous number of potential combinations of drugs with foods and beverages, it is not possible to evaluate them all in a human study. Thus, it would be interesting to determine the relationship between the results of the pediatric gustatory test and those obtained using the electronic tongue.

One limitation of this study is that subjects were not pediatric patients but healthy young adults. Nevertheless, subjects were considered to have a heightened sense of taste close to the pediatric population. Thus, the combinations with food or beverages described above are regarded as most likely recommended for pediatric patients in clinical settings. It would be of great interest to assess the acceptability of those combinations in such pediatric patients. Previously, parents have empirically selected the food and beverages to combine with powder formulations to administer to pediatric patients because of a lack of evidence. According to the results of this study, food and beverages affect palatability differently depending on the combination of formulations.

\section{CONCLUSION}

We quantitatively evaluated the palatability of powder formulations by performing the gustatory sensation test using VAS scores. The human gustatory sensation tests indicated that some foods and beverages improve the palatability of the powder formulations of azithromycin and carbocysteine. The results of this study indicate that ice cream improves the palatability of azithromycin, while yogurt improves the palatability of carbocysteine. Moreover, the subjects recommended these same combinations for pediatric patients. This study suggests that some foods and beverages improve the palatability of powder formulations, thereby decreasing the possibility that pediatric patients will refuse medications because of their unpleasant taste.

Acknowledgments The authors gratefully acknowledge Towa Pharmaceutical Co., Ltd. (Osaka, Japan) for donating the powder formulations of azithromycin and carbocysteine. We are grateful to Ms. Haruka Ogawa for her excellent technical assistance. We thank Melissa Crawford, Ph.D., for editing a draft of this manuscript.

Conflict of Interest The authors declare no conflict of interest.

\section{REFERENCES}

1) Klingmann V, Spomer N, Lerch C, Stoltenberg I, Frömke C, Bosse HM, Breitkreutz J, Meissner T. Favorable acceptance of mini-tablets compared with syrup: a randomized controlled trial in infants and preschool children. J. Pediatr., 163, 1728-1732.e1 (2013).

2) Klingmann V, Seitz A, Meissner T, Breitkreutz J, Moeltner A,
Bosse HM. Acceptability of uncoated mini-tablets in neonates-a randomized controlled trial. J. Pediatr., 167, 893-896.e2 (2015).

3) van Riet-Nales DA, de Neef BJ, Schobben AF, Ferreira JA, Egberts TC, Rademaker CM. Acceptability of different oral formulations in infants and preschool children. Arch. Dis. Child., 98, 725-731 (2013).

4) McCrindle BW, O’Neill MB, Cullen-Dean G, Helden E. Acceptability and compliance with two forms of cholestyramine in the treatment of hypercholesterolemia in children: a randomized, crossover trial. J. Pediatr., 130, 266-273 (1997).

5) European Medicines Agency. "Reflection paper: Formulations of choice for the paediatric population.”: 〈https://www.ema.europa. eu/en/documents/scientific-guideline/reflection-paper-formulationschoice-paediatric-population_en.pdf), accessed 5 May, 2020.

6) Ramgoolam A, Steele R. Formulations of antibiotics for children in primary care: effects on compliance and efficacy. Paediatr. Drugs, 4, 323-333 (2002).

7) Baguley D, Lim E, Bevan A, Pallet A, Faust SN. Prescribing for children-taste and palatability affect adherence to antibiotics: a review. Arch. Dis. Child., 97, 293-297 (2012).

8) Christopher-Paul Milne. The pediatric studies initiative. New Drug Development: A Regulatory Overview Eighth Edition. Vol. 1, Barnett Educational Services, Needham, pp. 339-362 (2005).

9) American Academy of Pediatrics. "Periodic Survey \#44 Patient Compliance with Prescription Regimens.”: 〈https://www.aap.org/en-us/ professional-resources/Research/Pages/PS44_Executive_Summary_ PatientCompliancewithPrescriptionRegimens.aspx.), accessed 5 May, 2020.

10) Sunakawa K, Akita H, Iwata S, Sato Y, Fujii R. Rational use of oral antibiotics for pediatric infections. Infection, 23 (Suppl. 2), S74-S78 (1995).

11) International Council for Harmonisation of Technical Requirements for Pharmaceuticals for Human Use. "ICH harmonised guideline addendum to ICH E11: Clinical investigation of medicinal products in the pediatric population E11 (R1).”: 〈https://database.ich.org/sites/ default/files/E11_R1_Addendum.pdf), accessed 5 May, 2020.

12) Pharmaceuticals and Medical Devices Agency. "Azithromycin hydrate (Zithromac).”: 〈https://www.pmda.go.jp/PmdaSearch/iyaku Detail/ResultDataSetPDF/671450_6149004C1030_2_12), accessed 5 May, 2020.

13) Pharmaceuticals and Medical Devices Agency. "L-Carbocisteine (Mucodyne-DS).”: ‘https://www.pmda.go.jp/PmdaSearch/iyakuDetail/ ResultDataSetPDF/230109_2233002R2029_1_04), accessed 5 May, 2020.

14) Nakamura $H$, Uchida $S$, Sugiura $T$, Namiki N. The prediction of the palatability of orally disintegrating tablets by an electronic gustatory system. Int. J. Pharm., 493, 305-312 (2015).

15) Takano H, Uchida S, Kashiwagura Y, Tanaka S, Hakamata A, Odagiri K, Inui N, Watanabe H, Namiki N. Preparation of cocoa powder-containing orally disintegrating tablets of rebamipide (rebamipide chocolet) and evaluation of their clinical palatability. Chem. Pharm. Bull., 67, 112-119 (2019).

16) Nakagaki F, Uchida $S$, Tanaka $S$, Namiki N. Palatability and preference of gummi formulations with various pharmaceutical characteristics. Chem. Pharm. Bull., 66, 452-457 (2018).

17) Nakano Y, Maeda A, Uchida S, Namiki N. Preparation and evaluation of unpleasant taste-masked pioglitazone orally disintegrating tablets. Int. J. Pharm., 446, 160-165 (2013).

18) Kimura S, Uchida S, Kanada K, Namiki N. Effect of granule properties on rough mouth feel and palatability of orally disintegrating tablets. Int. J. Pharm., 484, 156-162 (2015).

19) Matsui R, Uchida S, Namiki N. Combination effect of physical and gustatory taste masking for propiverine hydrochloride orally disintegrating tablets on palatability. Biol. Pharm. Bull., 38, 17-22 (2015)

20) Haefeli M, Elfering A. Pain assessment. Eur. Spine J., 15 (Suppl. 1), 
S17-S24 (2006).

21) Haraguchi $T$, Uchida $T$, Hazekawa $M$, Yoshida M, Nakashima $M$, Sanda H, Hase T, Tomoda Y. Ability of food/drink to reduce the bitterness intensity of topiramate as determined by taste sensor analysis. Chem. Pharm. Bull., 64, 14-20 (2016).

22) Uchida T, Tanigake A, Miyanaga Y, Matsuyama K, Kunitomo M, Kobayashi Y, Ikezaki H, Taniguchi A. Evaluation of the bitterness of antibiotics using a taste sensor. J. Pharm. Pharmacol., 55, 1479-1485 (2003).

23) Tanigake A, Miyanaga Y, Nakamura T, Tsuji E, Matsuyama K, Kunitomo M, Uchida T. The bitterness intensity of clarithromycin evaluated by a taste sensor. Chem. Pharm. Bull., 51, 1241-1245 (2003).

24) Haraguchi T, Okuno T, Nishikawa H, Kojima H, Ikegami S, Yoshida M, Habara M, Ikezaki H, Uchida T. The relationship between bitter taste sensor response and physicochemical properties of 47 pediatric medicines and their biopharmaceutics classification. Chem. Pharm. Bull., 67, 1271-1277 (2019).

25) Ishizaka T, Okada S, Takemoto E, Tokuyama E, Tsuji E, Mukai J, Uchida T. The suppression of enhanced bitterness intensity of macrolide dry syrup mixed with an acidic powder. Chem. Pharm. Bull., 55, 1452-1457 (2007). 